Musées, Patrimoine et Culture scientifiques et techniques

$165 \mid 2016$

mai-juin 2016

\title{
Le patrimoine géologique en France
}

Patrick De Wever, Grégoire Egoroff et Sylvain Charbonnier

URL : http://journals.openedition.org/ocim/1661

DOI : 10.4000/ocim. 1661

ISSN : 2108-646X

\section{Éditeur}

OCIM

Édition imprimée

Date de publication : 1 mai 2016

Pagination : $32-34$

ISSN : 0994-1908

Référence électronique

Patrick De Wever, Grégoire Egoroff et Sylvain Charbonnier, « Le patrimoine géologique en France », La Lettre de I'OCIM [En ligne], 165 | 2016, mis en ligne le 01 mars 2017, consulté le 20 avril 2019. URL: http://journals.openedition.org/ocim/1661; DOI : 10.4000/ocim.1661

Ce document a été généré automatiquement le 20 avril 2019

Tous droits réservés 


\title{
Le patrimoine géologique en France
}

\author{
Patrick De Wever, Grégoire Egoroff et Sylvain Charbonnier
}

Cet article (disponible sur www.ocim.fr, rubrique La Lettre de l'OCIM) mettait en lumière les différents outils mis en place pour repérer et décrire les objets géologiques remarquables.

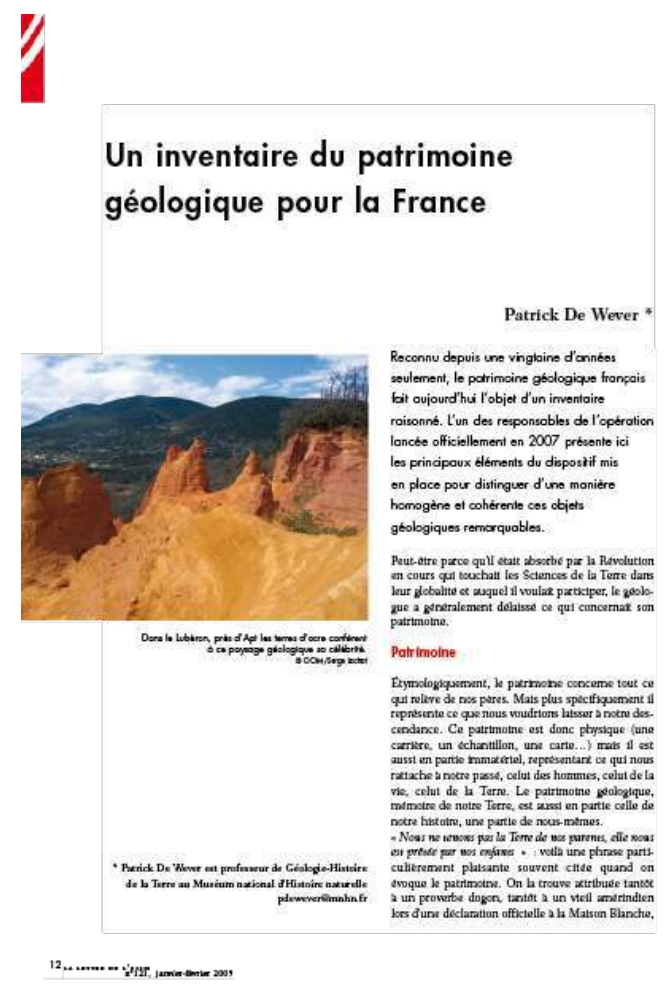

1 La notion de patrimoine naturel a longtemps revêtu les seuls aspects vivants de la nature : faune, flore, écosystèmes... Cet état de fait était souligné par l'usage d'un mot qui s'est imposé tout doucement, depuis 1992 : la biodiversité. Cette tendance a conduit à négliger longtemps ce qui est le socle de cette biodiversité : la géodiversité. En France, un premier virage est pris en 2002 avec la promulgation de la loi relative à la "Démocratie de proximité". En fait il s'agit plus précisément d'une nouvelle rédaction de l'article L411-5 
du Code de l'Environnement. Cette loi impose la réalisation d'un inventaire du patrimoine naturel, dont la géologie. Les procédures sont élaborées par une instance du ministère en charge de l'Environnement, la Conférence permanente du patrimoine géologique (CPPG) dans laquelle siègent des représentants des grands organismes, des associations professionnelles, des musées, et des amateurs. Le Muséum national d'Histoire naturelle (MNHN) en assure la responsabilité scientifique. L'inventaire est officiellement lancé en 2007 et un vade mecum en précise les modalités (De Wever et al., 2006), comme cela fut présenté en 2009 dans le n 121 de La Lettre de l'OCIM .

2 Les premiers inventaires ont été lancés avec un logiciel intitulé "Géotope", qui a été distribué aux instances compétentes, les données devant être récupérées au coup par coup. En région, certaines instances ont trouvé nécessaire de modifier légèrement le logiciel pour mieux répondre à certains de leurs besoins, de leurs souhaits. Il en est résulté que les données ne devenaient alors plus homogènes pour le territoire national, ce qui n'était évidemment pas sans poser problèmes. En 2010 le ministère décide donc de mettre au point un outil de saisie en ligne. Ce nouveau système a l'avantage de ne pas être modifiable localement et les données sont directement gérées au niveau national. Comme toute médaille a un revers, l'inconvénient associé est que l'outil a mis du temps pour être opérationnel facilement. Toujours est-il que l'inventaire avance, plus ou moins selon les régions, les départements. Un état d'étape a été organisé à Digne-les-Bains en 2012 pour que tous les acteurs puissent à la fois présenter leurs travaux et partager leurs expériences. Les actes de ces journées ont été publiés dans un mémoire spécial de la Société géologique de France (Egoroff et al., 2013).

\section{L'état de l'inventaire début 2016}

Le déroulement de l'inventaire s'effectue sur tout le territoire national, métropolitain ou ultra-marin à des vitesses différentes et avec un calendrier différencié. Certaines régions, certains départements s'y sont attelés précocement, d'autres plus tardivement. Toutes les "anciennes" régions l'ont entrepris sauf une. Il est cocasse de noter que la seule région qui n'ait pas lancé son inventaire selon les normes voulues par le ministère de l'Environnement soit l'ex-région Poitou-Charentes, qui fut longtemps administrée par l'actuelle ministre de l'Environnement !

4 Dans l'état des lieux que nous présentons sur la carte ci-contre nous avons choisi de retenir les départements comme entités administratives car ils présentent actuellement une plus grande stabilité que les régions et permettent aussi une présentation plus détaillée. Par ailleurs nous distinguons deux types de validations : validation régionale, étape obligatoire avant l'examen en validation nationale, comme le requiert le Loi de 2002. Les modalités ont été présentées dans le Vade Mecum publié en 2006 et précisées dans un autre mémoire spécial intitulé Géopatrimoine en France en 2014 (De Wever et al., 2006, 2014).

5 Actuellement 53 départements ont leur inventaire qui a été validé par les régions, plus précisément par les CSRPN (Conseil scientifique Régional du Patrimoine Naturel). 


\section{La place de l'inventaire national dans le concert international}

6 L'inventaire au niveau national était parfaitement compatible avec les autres inventaires nationaux de données naturelles tels que ceux des ZNIEFF (Zone Naturelle d'Intérêt Faunistique et Floristique) puisqu'il avait été voulu comme tel depuis le début. Néanmoins aujourd'hui il est impensable d'établir des données au niveau d'un seul pays, qui ne puissent être partagées au niveau international, au moins au niveau européen. Deux actions s'imposaient alors: d'une part comparer l'inventaire conduit en France avec les inventaires de quelques autres pays, d'autre part faire rencontrer les acteurs des inventaires de différents pays pour, certes effectuer des comparaisons, mais aussi partager des expériences sur les enjeux, les perspectives de ces inventaires. Une réunion internationale a alors été organisée en septembre 2015 : intitulée "Geoinv 2015 : enjeux, bilans, perspectives". Tenue au muséum d'Histoire naturelle de Toulouse, elle rassemblait des participants d'une vingtaine de pays. Cette réunion, organisée par le ministère de l'Environnement, le Muséum national d'Histoire naturelle et le muséum d'Histoire naturelle de Toulouse était placée sous l'égide de l'IUGS (International Union of Geological Sciences), l'UICN (Union Internationale pour la Conservation de la Nature) et l'UNESCO (Organisation des Nations unies pour l'éducation, la science et la culture). Elle a d'ailleurs bénéficié de la présence des présidents concernés de ces organismes prestigieux.

État de l'inventaire du patrimoine géologique francais par département

\section{Avancement}

Validé national

En révision

Examen mars 2016

En cours

Non lancé en 2015
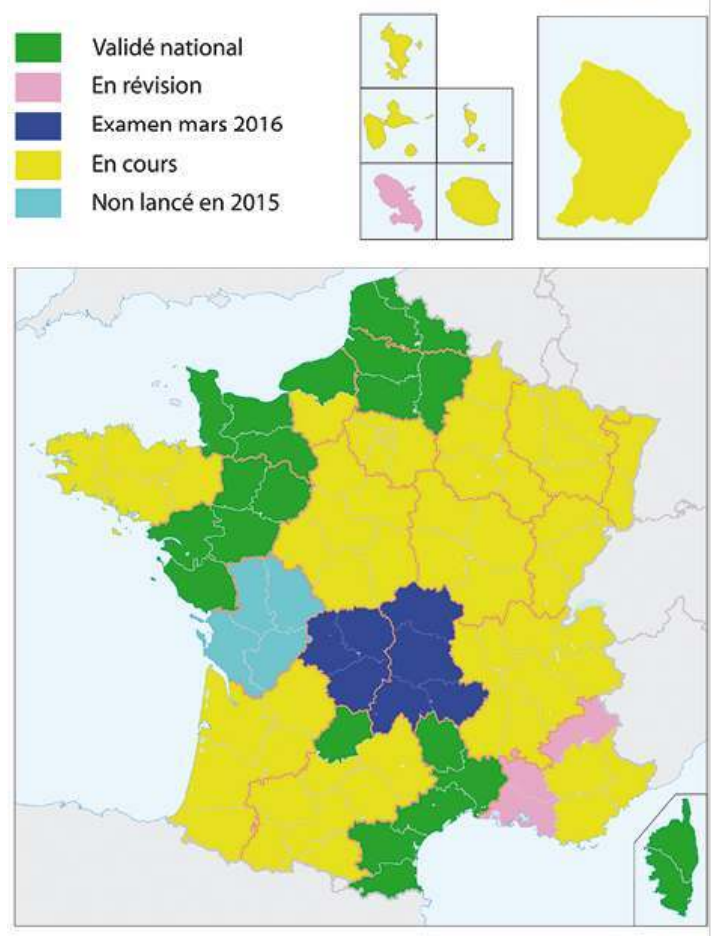

(c) Patrick De Wever/IGN 
7 La confrontation des inventaires de différents pays a révélé que sous un même mot des réalités bien différentes existaient. Certains petits pays (en superficie) par exemple affichent fièrement quelques milliers de sites dans leur inventaire terminé. Mais chaque site ne comporte que quelques lignes d'informations (Altério et al., 2014) à la différence de la France où l'inventaire semble certes être long à réaliser mais comporte un dossier vraiment informatif, en moyenne d'une dizaine de pages avec cartes, références bibliographiques, photographies...

8 Par ailleurs, l'inventaire ayant été conçu, dès l'origine, pour être compatible avec les autres données de nature, il suffit d'interroger une seule base de données pour avoir tous les éléments de nature (géologie, faune, flore, écosystèmes...) qui méritent d'être considérés sur une même carte.

\section{Un inventaire pour connaître, mais comment protéger?}

9 L'inventaire du patrimoine géologique a été lancé pour faire un état des lieux, pour connaître. Ce faisant des sites de première importance ressortent et certains apparaissant réellement en danger, requièrent une protection. Néanmoins, il existait là une impasse, car aucun statut légal ne permettait cette protection parfois très locale, et rapide. En effet les sites d'intérêt géologique qui pouvaient, jusqu'à présent, être protégés le pouvaient s'ils étaient inscrits dans quelques éléments de grande taille, tel un cœur de parc naturel, ou dans une réserve... tous gros dossiers à établir, qui demandent généralement des années d'instruction avant qu'une décision soit applicable. En clair il manquait une modalité qui permette à court terme de protéger, même des sites de petite taille, tel les APB (Arrêtés de Protection de Biotope) qui existent depuis des années pour des éléments vivants de Nature. Avec la mise en place de la SCAP (Stratégie de Création d'Aires protégées - Egoroff, 2011), ce vide juridique, identifié depuis des années, a été comblé fin 2015. En effet, l'annonce de la solution a été annoncée lors du congrès de Toulouse en septembre, et publiée au Journal officiel le 28 décembre 2015. Désormais un simple arrêté du Préfet permet de protéger un site, petit ou grand, qui contient des fossiles ou des minéraux, ou des structures géologiques remarquables.

\section{La géodiversité comme socle de la biodiversité}

10 Pour la nature la biodiversité a longtemps été considérée en tant que telle, certes pas indépendante de son écosystème; avec son vivant, mais sans tenir compte de son contexte géologique. Cette bizarrerie est d'autant plus étrange que certains organismes sont directement liés au substrat rocheux: les chamois n'ont pas les "pantoufles" des hippopotames car les rochers dans lesquels ils évoluent requièrent une forte pression au sol, à l'inverse du fond des fleuves. On ne trouve pas non plus des saules et des canards là où se développent les chênes et les lièvres, milieux différents fondamentalement par leur géologie. Mieux encore certains types de roches ne permettent pas aux arbres de se développer. Ainsi par exemple, partout dans le monde, les massifs de serpentinite sont nus. Les japonais leur donnent d'ailleurs le nom de Bozu Yama ( = "montagne bonze" moines peu chevelus, comme on le sait). 
11 Depuis peu, une prise de conscience semble se faire grâce à la sortie de publications scientifiques qui soulignent que la prise en compte de la géodiversité améliore la gestion de la biodiversité. Bref, même ceux qui ne considèrent que la biodiversité ont intérêt à prendre en compte la géodiversité. Nous ne donnons ici que quelques exemples.

12 L'intérêt pour la nature est généralement tellement restreint à sa partie vivante, que la Commission Européenne a trouvé nécessaire d'attirer l'attention des gestionnaires par une communication spécifique à l'été 2015 intitulée: "Geodiversity information enhances biodiversity conservation". Cette annonce s'appuie notamment sur une étude publiée dans Conservation Biology (Anderson et al., 2015) Le titre est explicite : les informations sur la géodiversité renforcent la conservation de la biodiversité.

13 Cet aspect sera aussi souligné, au niveau mondial cette fois, par le thème d'une session spécifique lors du prochain congrès géologique international à Cape-Town (août 2016), intitulé "geodiversity under pins biodiversity".

\section{Conclusion}

14 Le patrimoine géologique voit actuellement une explosion d'intérêts, tant pour le géotourisme, que pour l'enseignement: un master géopatrimoine est en cours d'élaboration au niveau européen. Il y a maintenant une récente et indubitable prise de conscience que la géodiversité est le socle de la biodiversité.

\section{BIBLIOGRAPHIE}

Alterio, I., De Wever, P., Egoroff, G. et Cornée, A. Quelques inventaires géologiques en Europe : une comparaison, Géochronique, $\mathrm{n}^{\circ}$ 131, septembre 2014, p. 19.

Anderson, M.-G., Comer, P.-J., Beier, P., Lawler, J.-J., Schloss, C.-A., Buttrick, S., Albano, C.-M. et Faith, D.-P. Case studies of conservation plans that incorporate geodiversity, Conservation Biology, vol. 29, n 3, pp. 680-691.

De Wever, P., Le Nechet, Y. et Cornée, A. Vade Mecum pour l'inventaire du patrimoine géologique, Mémoires de la Société géologique de France, Hors série n 12, 2006, 161 p.

De Wever, P. Un inventaire du Patrimoine géologique pour la France, La Lettre de l'OCIM, $\mathrm{n}^{\circ} 121$, 2009, pp. 12-18.

De Wever, P., Egoroff, G., Cornée, A. et Lalanne, A. (dir.) Géopatrimoine en France, Mémoires de la Société géologique de France, Hors série $\mathrm{n}^{\circ}$ 14, 2014, 180 p.

Egoroff, G., Cornée, A., De Wever, P. et Lalanne, A. (dir.) Actes du colloque Géopatrimoine, un lustre d'inventaire en France. 10-12 octobre 2012, Digne-les-Bains, Mémoires de la Société géologique de France, Hors série $\mathrm{n}^{\circ} 13,2013,218 \mathrm{p}$.

Egoroff, G., De Wever, P., Cornée, A. et Monod, K. Du Grenelle I à la protection du Patrimoine Géologique, Géochronique, n 119, 2011, p. 17. 


\section{RÉSUMÉS}

Initiée dans La Lettre de l'OCIM en 2015 à l'occasion des 30 ans de l'OCIM, la rubrique "Quoi de neuf ?" propose un retour sur un article marquant de l'histoire de la revue. À partir d'un corpus d'articles sélectionnés par la rédaction de La Lettre de l'OCIM, les membres du comité des Publications de l'OCIM ont sélectionné plusieurs contributions. Dans cette perspective, il a été demandé à l'auteur ou à un expert du domaine de revisiter la problématique exposée dans l'article à la lueur des changements intervenus, notamment dans les pratiques professionnelles, depuis son écriture et de proposer des éléments prospectifs sur la question.

Les auteurs font un retour sur l'article de Patrick De Wever Un inventaire du patrimoine géologique pour la France publié en 2009 dans le n' 121 de La Lettre de l'OCIM et qui présentait les principaux éléments de la démarche engagée pour la réalisation de l'inventaire du patrimoine géologique français.

INDEX

Mots-clés : patrimoine naturel, patrimoine géologique

\section{AUTEURS}

\section{PATRICK DE WEVER}

Géologue, professeur au Muséum national d'Histoire naturelle

pdewever@mnhn.fr

\section{GRÉGOIRE EGOROFF}

ingénieur au Muséum national d'Histoire naturelle

geopatrimoine@mnhn.fr

\section{SYLVAIN CHARBONNIER}

géologue, maître de conférences au Muséum national d'Histoire naturelle

scharbonnier@mnhn.fr 